PATHOLOGY

doi: $10.1590 / \mathrm{S} 1677-55382010000700021$

\title{
Application of the Epstein criteria for prediction of clinically insignificant prostate cancer in Korean men
}

Lee SE, Kim DS, Lee WK, Park HZ, Lee CJ, Doo SH, Jeong SJ, Yoon CY, Byun SS, Choe G, Hwang SI, Lee HJ, Hong SK

Department of Urology, Seoul National Bundang Hospital, Seongnam, Korea

BJU Int. 2010; 105: 1526-30

Objective: To investigate the rate of pathologically confirmed unfavourable prostate cancers among Korean men who fulfilled the contemporary Epstein criteria for clinically insignificant prostate cancer.

Patients and Methods: This was a retrospective study of 131 Korean men who underwent radical prostatectomy (RP) for clinically insignificant prostate cancer as defined by contemporary Epstein criteria. We assessed the percentage of unfavourable prostate cancer (pathological Gleason sum $>$ or $=7$ and/or extraprostatic extension [EPE]) among these men and tried to identify useful predictors for such unfavourable tumour profiles using uni- and multivariate analyses.

Results: Among 131 men with clinically insignificant prostate cancer, $40(30.5 \%)$ had pathological Gleason $>$ or $=7$ tumours after RP. Of these 40 men, four (3.1\%) also had EPE on examination of RP specimen. All those who did not have Gleason score upgrading after RP had organ-confined disease from examination of RP specimen. Overall, $40(30.5 \%)$ of the 131 men who fulfilled the contemporary Epstein criteria for clinically insignificant prostate cancer before RP had pathologically unfavourable disease. Among our patients, no significant preoperative predictor of pathologically unfavourable disease was identified using uni- and multivariate analyses.

Conclusion: Our results showed that a significant proportion of contemporary Korean patients who meet all the conditions of the contemporary Epstein criteria for prediction of clinically insignificant prostate cancer might actually harbour prostate cancer with unfavourable pathological features. Such findings should be considered when treatment options are contemplated based upon the Epstein criteria among Asian patients.

\section{Editorial Comment}

Watchful waiting (active surveillance) has gaining popularity as a management strategy for newly diagnosed low-grade, limited cancer on needle biopsy based on the large discrepancy between incidence and mortality rate of prostate cancer. Data from the European Randomized Study of Screening for Prostate Cancer Trial showed that PSA screening reduced the rate of death from prostate cancer by $20 \%$ but was associated with a high risk of overdiagnosis (1).

Epstein proposed a set of criteria based on PSA and preoperative biopsy features that identify a high likelihood of organ-confined insignificant cancers at radical prostatectomy (2). Insignificant cancers are defined as cancers confined to the prostate (pT2), Gleason low-grade (score $\leq 6)$, and low-volume ( $\leq 0.5 \mathrm{cc})$. It is important to be aware that insignificant cancer is not synonymous of latent (indolent) carcinoma. So far, there is no single marker of biological behavior for prostate cancer.

The preoperative biopsy and clinical features of Epstein's criteria for insignificant cancer are: clinical stage T1c, preoperative PSA density $<0.15$, no Gleason pattern 4 or 5 , no more than 2 cores with cancer, and no more than $50 \%$ of involvement of cancer in a single core. According to Bastian at Johns Hopkins, the predictive value using the Epstein's criteria for organ-confined disease and insignificant cancer is $92 \%$ and $84 \%$, respectively.

Several studies have applied Epstein's criteria for prediction of clinically insignificant prostate cancer with differing results. In all studies, the criteria are predictive of a high frequency of organ-confined disease 
but differ for insignificant cancer. In the study from Korea, the frequency of organ-confined disease was $97 \%$ (vs. 92\% from Johns Hopkins) but 69\% for insignificant cancer (vs. 84\% from Johns Hopkins).

\section{References}

1. Schröder FH, Hugosson J, Roobol MJ, Tammela TL, Ciatto S, Nelen V, et al.: Screening and prostate-cancer mortality in a randomized European study. N Engl J Med. 2009; 360: 1320-8.

2. Bastian PJ, Mangold LA, Epstein JI, Partin AW: Characteristics of insignificant clinical T1c prostate tumors. A contemporary analysis. Cancer. 2004; 101: 2001-5.

Dr. Athanase Billis

Full-Professor of Pathology

State University of Campinas, Unicamp

Campinas, São Paulo, Brazil

E-mail: athanase@fcm.unicamp.br

doi: 10.1590/S1677-55382010000700022

\section{The Epstein criteria predict for organ-confined but not insignificant disease and a high likelihood of cure at radical prostatectomy}

Lee MC, Dong F, Stephenson AJ, Jones JS, Magi-Galluzzi C, Klein EA

Glickman Urological and Kidney Institute, Cleveland Clinic, Cleveland, OH, USA

Eur Urol. 2010; 58: 90-5

Background: Few reports attempt to validate the role of Epstein criteria in selecting patients for an active surveillance protocol.

Objective: To determine the performance of the Epstein biopsy criteria for predicting pathologic end points and biochemical relapse-free survival (bRFS) in men with early stage prostate cancer (PCa) treated by radical prostatectomy (RP).

Design, Setting and Participants: Between October 1999 and January 2007, 746 consecutive patients were biopsied, and then underwent RP at our tertiary care institution. Two hundred sixty-eight patients met the entry criteria of Gleason 6 disease only on initial biopsy with complete pathologic information.

Measurements: Primary end point was insignificant disease. Insignificant disease was defined using a classical (organ-confined, Gleason score $<6$, and tumor volume $<0.5 \mathrm{~cm}(3)$ ) and more liberal (organ-confined, Gleason $<6$ tumor of any volume) formulation. Secondary end points included organ-confined disease and bRFS.

Results and Limitations: One hundred thirty-six men (51\%) met the Epstein biopsy criteria, and 167 (62\%) had organ-confined cancer. Insignificant disease by the classical and liberal definitions was present in $68(25 \%)$ and 92 (34\%) patients, respectively. Cases meeting Epstein biopsy criteria were more likely to have insignificant disease by either definition $(\mathrm{p}<0.001)$ and more likely to have organ-confined tumors $(\mathrm{p}<0.001)$. Sensitivity, specificity, positive predictive value (PPV), and negative predictive value (NPV) varied widely among the end points, with sensitivity (74\%) and NPV (86\%) best for the classical definition of insignificant disease and specificity (74\%) and PPV (92\%) best for organ-confined disease. The estimated 5-yr bRFS was 100\% for those meeting Epstein biopsy criteria compared to $83 \%$ for those not meeting these criteria.

Conclusions: The Epstein biopsy criteria predict for a high likelihood of organ-confined disease and the absence of biochemical failure up to 5 years after RP. These criteria are insufficiently robust to predict the presence of biologically insignificant disease. 